Supporting Information

for

\title{
Probing Magnetism in Artificial Metal-Organic Complexes Using Electronic Spin Relaxometry
}

\author{
Xue Zhang ${ }^{1,2,+}$, Philip Willke ${ }^{1,2,+}$, Aparajita Singha ${ }^{1,2}$, Christoph Wolf $^{1,2}$, Taner Esat ${ }^{1,2}$, Minhee \\ Choi $^{1,3}$, Andreas J. Heinrich ${ }^{1,3}$ and Taeyoung Choi ${ }^{1,3}{ }^{*}$ \\ 1 Center for Quantum Nanoscience, Institute for Basic Science (IBS), Seoul 03760, Republic \\ of Korea \\ 2 Ewha Womans University, Seoul 03760, Republic of Korea \\ 3 Department of Physics, Ewha Womans University, Seoul 03760, Republic of Korea
}

*E-mail: choi.taeyoung@qns.science

\section{Sample preparation and experiment conditions}

Atomically flat and clean $\mathrm{Ag}(100)$ surface were obtained by alternative annealing and $\mathrm{Ar}^{+}$ sputtering cycles. To grow $\mathrm{MgO}$, magnesium was evaporated thermally onto clean $\operatorname{Ag}(100)$ which was kept at $\sim 400{ }^{\circ} \mathrm{C}$ in an oxygen atmosphere of $1.0 \times 10^{-6}$ torr. The thickness of $\mathrm{MgO}$ was identified by point-contact measurement and was two monolayers in this work. Commercial TCNE with $>98 \%$ purity was sublimated onto $\mathrm{MgO} / \mathrm{Ag}(100)$ from a home-made container by pumping TCNE to $\sim 1.5 \times 10^{-8}$ torr, close to its saturated vapor pressure. Single $\mathrm{Fe}$ atoms were evaporated after TCNE. For both Fe and TCNE deposition, the sample was kept at $\sim 30 \mathrm{~K}$. All measurements in our work were carried out at $2 \mathrm{~K}$ and in an out-of-plane magnetic field of $2 \mathrm{~T}$. Pt/Ir tip was used and treated by poking gently into bare $\operatorname{Ag}(100)$ surface before picking up $\mathrm{Fe}$ atoms to make it spin-polarized. Scanning and pump-probe bias was applied to the tip while sample was grounded.

\section{STM manipulation process of building a Fe(TCNE) complex}

Referring to Figure 1(e), i) we position the tip on top of an isolated TCNE and increase the bias stepwise from $0.6 \mathrm{~V}$ at the set current of $40 \mathrm{pA}$; ii) when the bias is large enough ( $\sim 900$ $\mathrm{mV}$ ), the TCNE preferentially hops onto the tip apex and this generates a drastic change in the tunneling current and vertical tip position (left black arrow in Figure 1(f)). Then we disable the feedback loop, retract the tip by a few angstroms and lower the bias simultaneously (to $\sim 3 \mathrm{mV}$ ) to avoid further molecule hopping; iii) we move the tip on top of a single $\mathrm{Fe}$ atom and approach towards the $\mathrm{Fe}$ atom in open loop; iv) we retract the tip when sudden current drop occurs which indicates the falling off of TCNE (right black arrow in Figure 1(f)) and contacting to Fe atom.

\section{Electronic properties of TCNE on MgO}

TCNE molecules are weakly bonded to the MgO surface. They can easily move or hop under larger scanning bias and tunneling current which makes it difficult to measure the molecular electronic properties. Figure $\mathrm{S} 1$ shows the $\mathrm{d} I / \mathrm{d} V$ and $I-V$ measurements of a single TCNE adsorbed on $\mathrm{MgO}$ within a small bias window. In our experiment setup, the tip voltage is biased while the sample is grounded. Thus, Figure $\mathrm{S} 1$ reveals an onset of HOMO at $\sim 1 \mathrm{~V}$ for 
TCNE on MgO. LUMO is not detected due to the molecular instability when a bias voltage below $-1.2 \mathrm{~V}$ is applied.

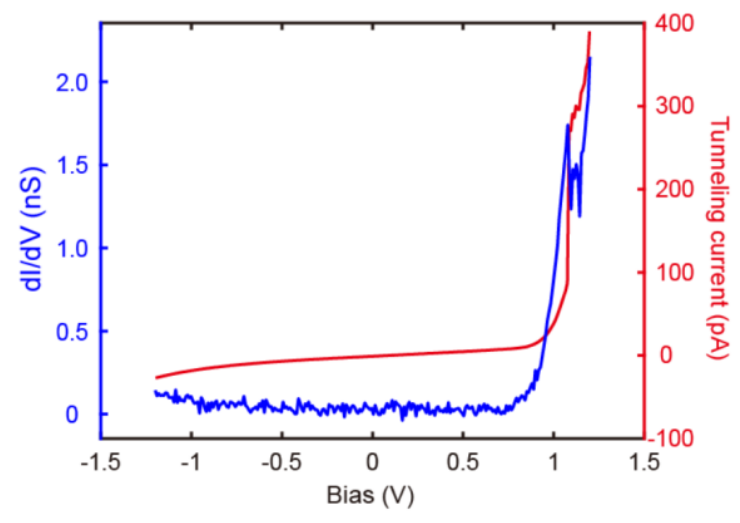

Figure S1. Electronic properties of isolated TCNE on MgO. Blue: $\mathrm{d} / / \mathrm{d} V$ spectrum measured with $30 \mathrm{mV}$ modulation voltage. The tunneling condition for the spectroscopy was set at $V=-1.2 \mathrm{~V}, I=$ $25 \mathrm{pA}$ before disabling the feedback loop. Red: Simultaneously recorded tunneling current while ramping bias.

\section{Statistics of spin-IETS measured on different complexes}

In our work, we have built 32 complexes reproducibly based on the manipulation process shown in Figure 1(e,f) and observed that the topographic appearances are similar to each other in that the topographic height of the Fe atoms reduces significantly. The STM topography of each complex may show subtle differences due to the variety of TCNE orientations and the location of the Fe with respect to the TCNE. For all complexes, we measured the spin-IETS with the tip positioned roughly over the Fe locations before complex formation. Most of the complexes indicate the spin excitation energies at $\sim 14.6 \mathrm{mV}$, as shown in Figure S2(a), which is similar to the one measured for isolated $\mathrm{Fe}$ atoms under the same experimental conditions. Some complexes have a smaller spin excitation energy at around 11-12 mV. However, these complexes are much fewer in numbers. Figure S2(b) and (c) display two types of Fe(TCNE) complexes (type I and II), represented by complex \#1 and \#3, respectively. Compared to isolated Fe atoms, the energy of the spin-IETS feature for the type I complex (Figure S2(b)) is smaller while for the type II complex (Figure S2(c)) it is comparable. The topographic images for type I and II are also slightly different. Type I is characterized by a faint bump located out of dim halo and the distance between the faint bump and the center of TCNE is $\sim 0.57 \mathrm{~nm}$. For type II, there is usually a faint bump inside the dim halo which is $\sim 0.45 \mathrm{~nm}$ away from the center of TCNE. We note that the type I complex can be readily switched to the type II complex by tip perturbation (Figure S12). As shown in Figure 3 and S5, we do not find a significant change in the $T_{1}$ for type I and II complexes. 
(a)

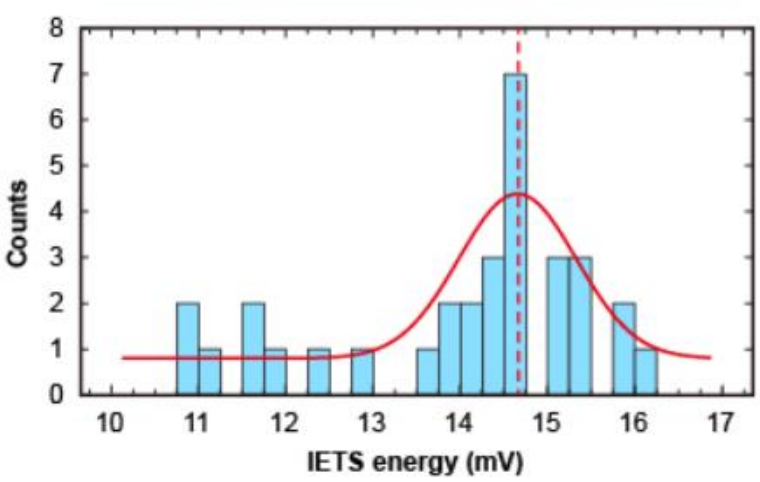

(b)

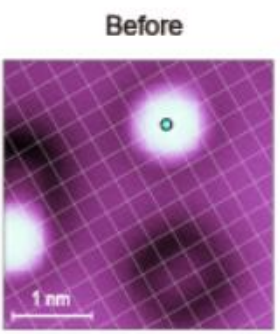

(c)

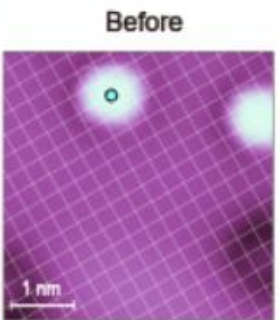

After

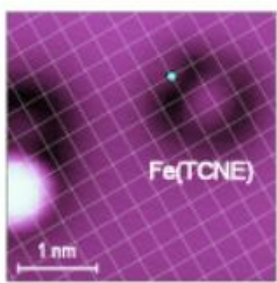

After

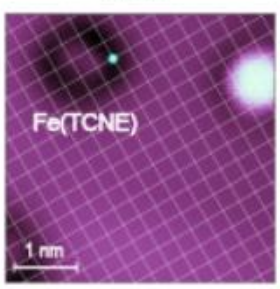

Type I: narrower spin-IETS

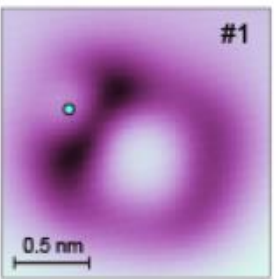

Type II: comparable spin-IETS

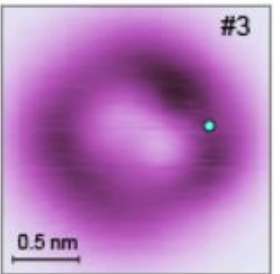

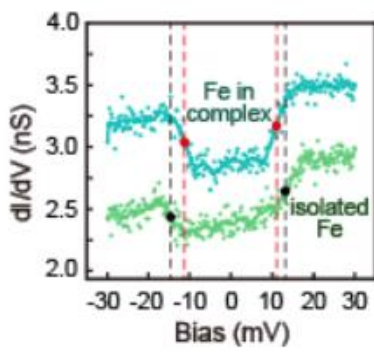

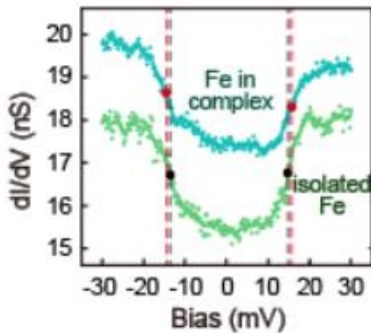

Figure S2. Statistics of spin-IETS features measured on different Fe(TCNE) complexes. (a) Histogram of the spin-IETS energies of different complexes. The red curve originates from a Gaussian fit to the histogram, which yields a mean value of $14.6 \mathrm{mV}$ (dashed red line). (b) Construction process and $\mathrm{d} I / \mathrm{d} V$ spectrum of a complex (labeled as \#1 in the main text) showing a narrower IETS step at $\sim 11 \mathrm{mV}$. The $\mathrm{d} / / \mathrm{d} V$ of the Fe in the complex is shifted upwards by $0.6 \mathrm{nS}$ for clarity. (c) A representative complex (labeled as \#3 in the main text) showing an IETS step comparable to one of an isolated $\mathrm{Fe}$ atom. The $\mathrm{d} / / \mathrm{d} V$ of the $\mathrm{Fe}$ in the complex is shifted upwards by $1.8 \mathrm{nS}$ for clarity. The crossing points of the white grids represent oxygen sites of the $\mathrm{MgO}$. All STM images were acquired in constant-current mode and using (a) left: $V=-80 \mathrm{mV}, I=50 \mathrm{pA}$; middle: $V=-80 \mathrm{mV}, I=60 \mathrm{pA}$, right: $V=30 \mathrm{mV}, I=80 \mathrm{pA}$. (b) left: $V=-0.2 \mathrm{~V}, I=100 \mathrm{pA}$, middle: $V=-0.1 \mathrm{~V}, I=100 \mathrm{pA}$, right: $V=-0.1 \mathrm{~V}, I=100 \mathrm{pA}$. For $\mathrm{d} I / \mathrm{d} V$ in (a) and (b), the tip was placed at $V=30 \mathrm{mV}, I=80 \mathrm{pA}$ and $V=-30 \mathrm{mV}, I=500 \mathrm{pA}$, respectively before disabling the feedback loop. The modulation voltages used in (a) and (b) were $3 \mathrm{mV}$ and $1.5 \mathrm{mV}$, respectively.

\section{5. $\mathrm{d} I / \mathrm{d} V$ spectroscopy of the $\mathrm{Fe}$ in a $\mathrm{Fe}(\mathrm{TCNE})$ complex measured with a spin-polarized STM tip}

To verify the magnetic origin of the step-like feature in the $\mathrm{d} I / \mathrm{d} V$ spectra, a spin-polarized (SP) tip was used in $\mathrm{d} I / \mathrm{d} V$ measurements. While a non-SP tip detects symmetrical IETS steps at the same positions as for isolated $\mathrm{Fe}$ atoms, the spin-polarized $\mathrm{d} / / \mathrm{d} V$ exhibits a rather 
pronounced asymmetry which is related to spin polarized tunneling channels and spin pumping. ${ }^{37}$ This is shown for the Fe(TCNE) complex \#1 in Figure S3. We note that the spin contrast can vary at different positions on the complex, as indicated from the $\mathrm{d} I / \mathrm{d} V$ spectra shown in Figure S3(c).

(a)

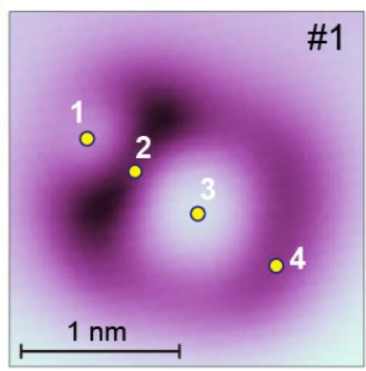

(b)

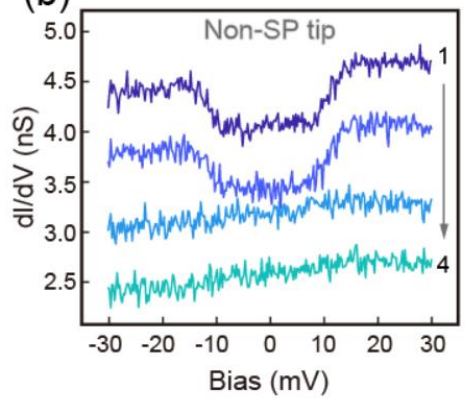

(c)

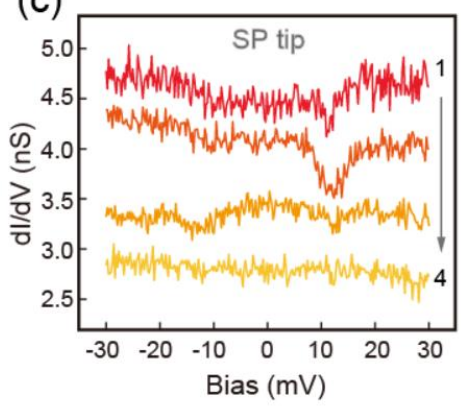

Figure S3. $\mathrm{d} I / \mathrm{d} V$ spectra measured at different positions on the (a) Fe(TCNE) complex \#1 with (b) a non-SP tip and (c) a SP tip respectively. The spectra are shifted vertically from one another by 0.6 $\mathrm{nS}$ (bottom to up). The tunneling conditions for both $\mathrm{d} I / \mathrm{d} V$ spectroscopies were $V=30 \mathrm{mV}, I=80$ pA before disabling feedback loop. $V_{\bmod }=3 \mathrm{mV}$.

\section{Pump-probe technique}

The spin relaxation time detection in this work follows the electronic pump-probe method reported in Ref. 19. An arbitrary wavefunction generator (Tektronix AWG 70002A) was connected to the tip though semi-rigid coaxial wires to send serial alternating pump and probe voltage pulses. Pump voltage in this work was $95 \mathrm{mV}$, high enough to excite the spin from its ground state to the first excited state by means of the transitions to other states since the direct transition between the ground state and the first excited state is negligible. ${ }^{20}$ Schematic energylevel diagram of Fe spin states is shown in Figure S4. Probe voltage used here, $5 \mathrm{mV}$, was rather low to avoid further excitation during the spin relaxation process. Nevertheless, the small probe voltage is sufficient to generate spin-polarized tunneling and detect the immediate spin state. The tunneling current of the probe pulse was measured by lock-in amplifier selectively detecting the $93 \mathrm{~Hz}$ component of the current. No bias was applied between pump and probe pulses so that spontaneous relaxation from the excited state and ground state could occur freely. The time interval between pump and probe pulses (delay time, $\Delta t$ ) was swept to get information of dynamical spin evolution. More details about the delay time maps are provided in Supporting Information Section 8. 


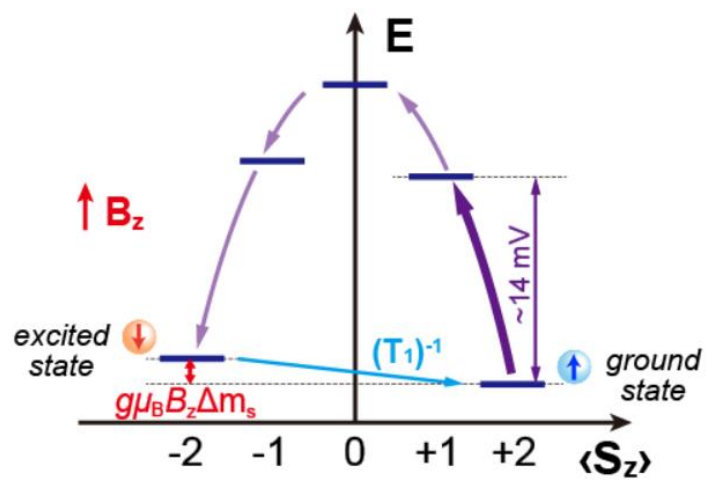

Figure S4. Energy-level diagram of the spin states of an isolated Fe atom. The red double arrow depicts the Zeeman splitting of the two lowest energy levels when a magnetic field along the easyaxis of the $\mathrm{Fe}$ spin is applied. The light blue arrow indicates the energy relaxation from the metastable excited state to the ground state, which can be measured by pump-probe spectroscopy.

\section{7. $T_{1}$ as a function of tunneling conductance on different complexes}

We performed pump-probe measurements with varying tunneling conductance on additional Fe(TCNE) complexes (\#1-\#5), as shown in Figure S5. All of the complexes show consistently a lowered $T_{1}$ compared to the $T_{1}$ of the isolated Fe. We can fit our experimental data by using an inelastic electron scattering model. ${ }^{20}$ When we extrapolate the fitted curves in the low conductance regime (tip-sample conductance $G_{t s}<10^{-1} \mathrm{nS}$ ), we find that the $T_{1}$ values become saturated to the millisecond range. This implies that the $T_{1}$ of the complexes is mainly limited by substrate scattering and this substrate-limited intrinsic $T_{1}$ is of the same order of magnitude as the isolated $\mathrm{Fe}$ atom. Here, we rewrite the fitting formula Eq. (1) as below,

$$
T_{1}=\frac{T_{1}^{0}}{\left(1+\frac{G}{G_{S S}}\right)^{2}}
$$

where $T_{1}^{0}$ is the intrinsic spin lifetime limited by the substrate scattering and $G_{S S}$ is the conductance contribution of substrate electrons scattering off the Fe spin and back to the substrate. $T_{1}^{0}$ and $G_{s S}$ are fitting parameters in our case. The intrinsic spin lifetime of an isolated $\mathrm{Fe}$ atom and the conductance contribution of substrate-substrate scattering on an isolated Fe spin have been reported in Ref. 20.

For fitting the experimental data, we use an upper limit for $T_{1}^{0}$ of $10 \mathrm{~ms}$. This was previously found to be roughly the spin lifetime limit for isolated Fe atoms set by non-electronic relaxation when the tip is withdrawn ${ }^{20}$. The results of the fits for $T_{1}^{0}$ and $G_{s S}$ are shown in Table S1 for the different complexes and an isolated Fe atom. Interestingly, we find that the conductance contribution of substrate electron scattering in the case of the Fe(TCNE) complexes seems weaker than in the case of an isolated Fe atom, since $G_{S S}$ for the complexes are lower than for the isolated Fe atom in our work. One likely reason is that the number of conductance channels is reduced due to the charge transfer between the Fe atom and TCNE. The deviation in the sample-sample conductance for our isolated Fe atom compared to Ref. 20 ( $\sim 4.5 \mathrm{nS}$ for $2 \mathrm{ML} \mathrm{MgO}$ ) likely results from a difference in the local morphological structure of $\mathrm{MgO}$ layers, which may also cause a slight change in the $T_{1}$ of a complex. 
Table S1 Fitting parameters of Eq. (S1)

\begin{tabular}{c|cccccc}
\hline & $\# 1$ & $\# 2$ & $\# 3$ & $\# 4$ & $\# 5$ & $\mathrm{Fe}$ \\
\hline$T_{1}^{0}(\mathrm{~ms})$ & 0.17 & 9.92 & 9.92 & 1.61 & 9.92 & 9.92 \\
\hline$G_{s s}(\mathrm{nS})$ & 0.80 & 0.20 & 0.17 & 0.33 & 0.21 & 1.47 \\
\hline
\end{tabular}
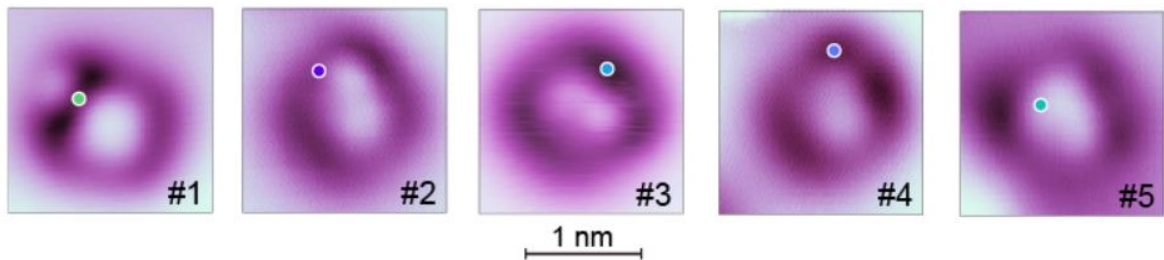

Tunneling current $I(\mathrm{pA})$

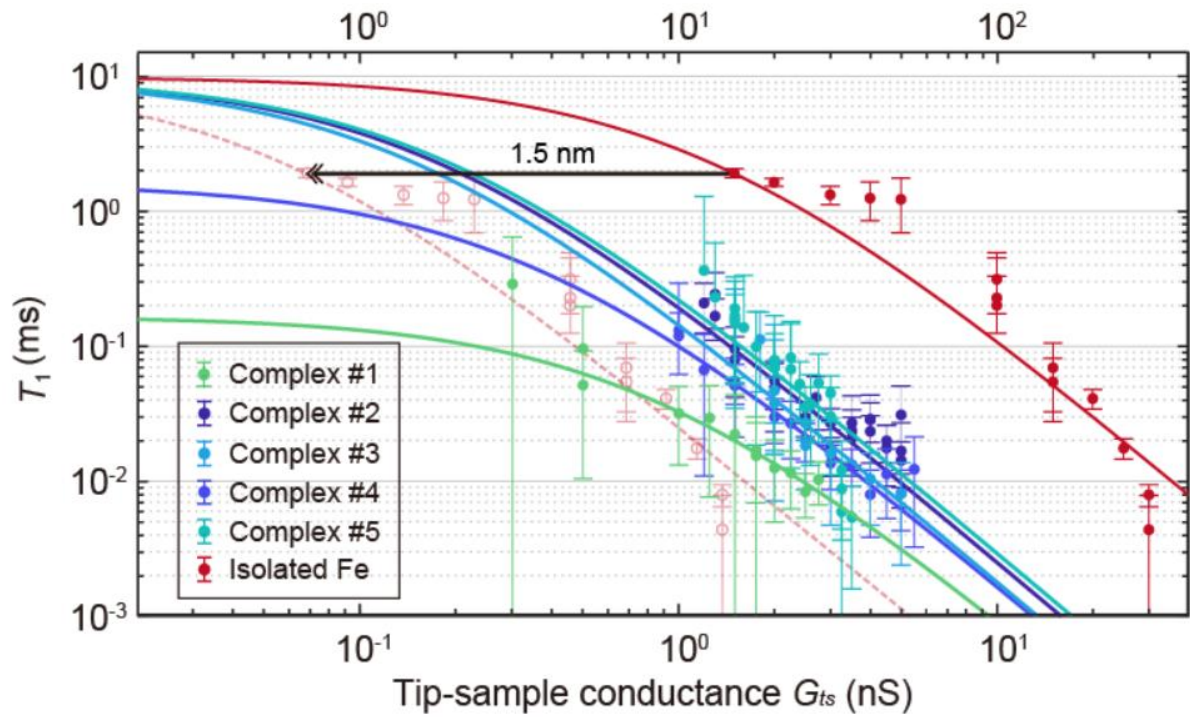

Figure S5. Top panel: STM images of different Fe(TCNE) complexes built in the same way as Figure 1. The colored spots indicate where the pump-probe measurements are performed. Lower panel: $T_{1}$ as a function of tunneling conductance measured on different complexes and an isolated $\mathrm{Fe}$ atom. The $T_{1}$ values and error bars are obtained from exponential fits to pump-probe data ( $T_{1}$ of isolated Fe at $I<100 \mathrm{pA}$ is measured by two-state switching method). Solid lines are the fits based on Eq. (S1). The dashed red line and open red circles are from the horizontal shift of the isolated Fe dataset by a distance corresponding to $1.5 \mathrm{~nm}$.

\section{Delay time maps of $\mathrm{Fe}(\mathrm{TCNE})$ complexes}

We find a shorter lifetime when the tip is closer to the assumed position of the Fe atom, as shown in Figure 4(a) and (b). Figure S6 displays the error bars of these fitted $T_{1}$ at different spots. At the black spot, the $T_{1}$ is much shorter than our time-resolution of this measurement and therefore no exponentially decaying signal is obtained. 

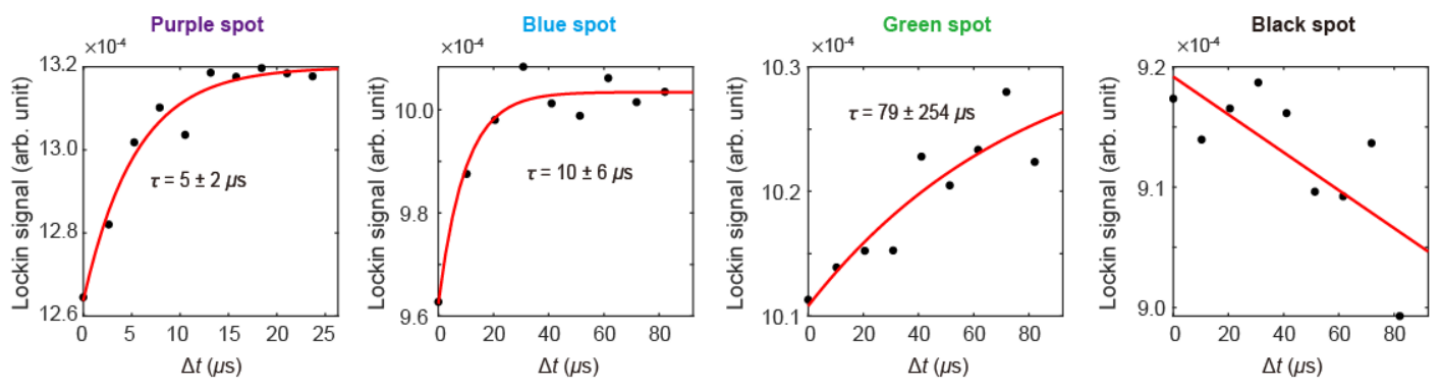

Figure S6. Fitted $T_{1}$ values and their corresponding error bars of the representative pump-probe data shown in Figure 4.

In the pump-probe experiment, we record the difference in tunneling currents $\Delta I=$ $I\left(\Delta t_{n}\right)-I\left(\Delta t_{0}=0\right)$ between a fixed pump-probe delay time $\left(\Delta t_{n}\right)$ and no delay time $\left(\Delta t_{0}=\right.$ 0 ) at each position of the complex while maintaining the same tunneling condition. This means that when the spin lifetime measured within the complex is similar to $\Delta t_{n}$, one can observe the difference in current $(\Delta I)$ (yellow and green area in Figure S7). On the other hand, when the measured lifetime is much faster or slower than $\Delta t_{n}$, one can see no difference in current due to low spin contrast or $T_{1}$ being too long (blue area in Figure S7). This allows us to spatially map out the spin lifetime across the complex.

At a fixed tip position over the complex, we first perform a pump-probe measurement with the feedback loop disabled. After the pump-probe measurement is done, we engage the feedback loop with the same tunneling parameters as before and the tip moves over the next spot and we start another pump-probe measurement. Thus, the tip-sample conductance remains constant for the pump-probe measurement of each spot above the complex.

Figure S7 shows the list of the delay time maps acquired with different $\Delta t_{n}$ on complex \#1. These pump-probe maps are characterized by a ring-like shape that grows in size for increased delay times $\left(\Delta t_{n}\right)$. As the tip moves laterally away from the spin center within the complex, the spin scattering between the spin center and the tip reduces and this makes the lifetime of the spin center longer, resulting in the ring-like shape, which grows in size for longer delay times. When the tip is located too far away $(>0.5 \mathrm{~nm})$ from the spin center, the spin sensitivity from the tip becomes too weak to measure the pump-probe signal. On the other hand, when the tip is closer to the spin center, the lifetime of the spin center becomes too short to measure the difference in current $(\Delta I)$. In addition, we show the spatial $T_{1}$ map based on the fits of pump-probe measurements across the complex (the bottom right panel of Figure S7). Therefore, these measurements intuitively illustrate the concentric spatial growth of the ring for increasing $\Delta t$ and provide a reliable criterion to locate the position of the nearly vanished $\mathrm{Fe}$ (in the area) with the lowest $T_{1}$.

Mapping out the spatial spin relaxation of another complex (\#5) verifies the versatility of this method (Figure S8). However, the $T_{1}$ map is not accessible here because no reliable $T_{1}$ can be fitted based on only two delay time maps. 


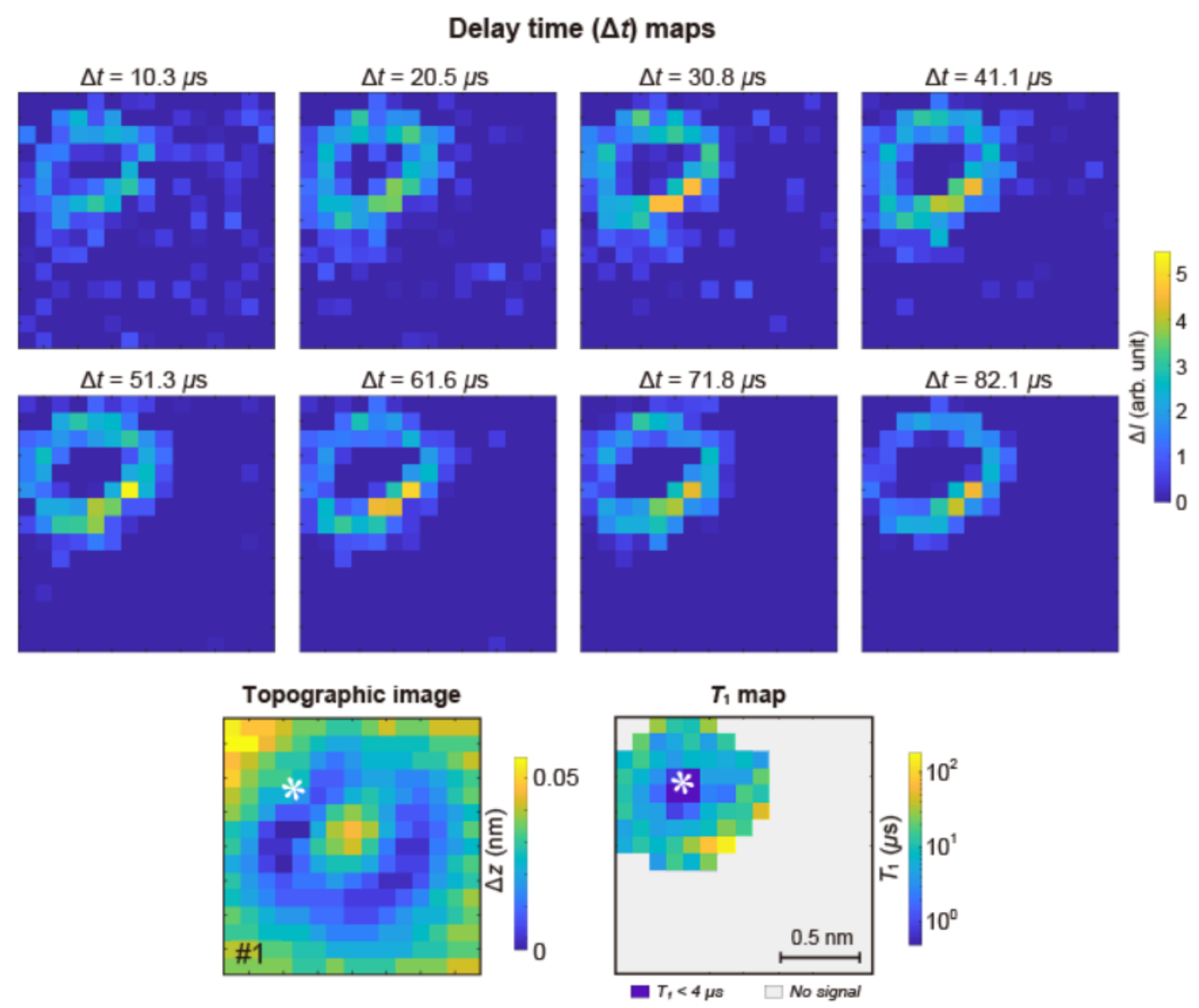

Figure S7. Delay time maps of Fe(TCNE) complex \#1. The estimated Fe position is marked by a white star in both topographic image and $T_{1}$ map.

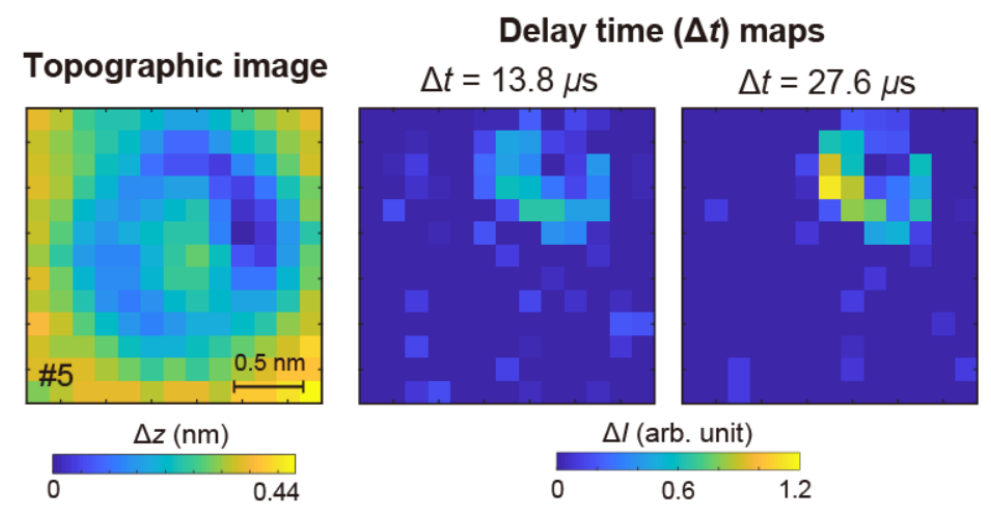

Figure S8. Delay time maps of complex \#5. Tip was placed at $V=10 \mathrm{mV}, I=30 \mathrm{pA}$ before performing spatial pump-probe imaging.

\section{Simulation of spatially resolved $T_{1}$ measurements}

To simulate the spatial map of the $T_{1}$ measurement across the Fe(TCNE) complex, we used the scattering model established in Ref. 20. Here, the relaxation rate $r_{T_{1}}$ is given by 


$$
r_{T_{1}}=\frac{1}{T_{1}}=P \frac{E_{01}}{e^{2}}\left(G_{t t}+2 \sqrt{G_{s S} G_{t t}}+G_{s S}\right)
$$

Here, $P$ is the probability of an electron scattered off by the Fe spin inelastically, $E_{01}$ is the energy splitting between the ground state and the first excited state given by the Zeeman splitting $E_{01}=2 \mu_{F e} B$ (we use the magnetic moment of $\mathrm{Fe} \mu_{F e}=5.4 \mu_{B}$ found in previous works ${ }^{22,38}$ ) and $e$ is the electron charge. Also note, that Eq. (S2) is a slightly rewritten form of Eq. (S1).

In the absence of the tip, $G_{S S}$ will only contribute to $T_{1}$, which is in the millisecond range under these conditions. ${ }^{20}$ When we take account for tip-tip scattering, $T_{1}$ shortens to tenth of microseconds as shown in our measurement. Thus, in this range $G_{t t}$ dominates, enabling us to approximate Eq. (S2) as

$$
r_{T_{1}} \approx P \frac{E_{01}}{e^{2}} G_{t t} .
$$

Moreover, the tip-tip conductance can be expressed as $G_{t t}=\exp \left(-2 k_{t} z_{t}\right)^{20}$ where $k_{t}$ is the tunneling electron decay rate over distance $z_{t}$ on the tip side. For the sample-sample side a decay rate of $k_{s} \sim 11.5 \mathrm{~nm}^{-1}$ (decay through the $\mathrm{MgO}$ layers to the Ag substrate) was previously determined. ${ }^{20}$ We generalize the distance $z_{t}$ as $d=$ $\sqrt{\left(x-x_{0}\right)^{2}+\left(y-y_{0}\right)^{2}+\left(z-z_{0}\right)^{2}}$ to also account for the lateral position in the map. Here, $\left(x_{0}, y_{0}, z_{0}\right)$ refer to the actual position of $\mathrm{Fe}$ spin center. This leads to the rate equation in its final form

$$
r_{T_{1}}(x, y, z) \approx P \frac{E_{01}}{e^{2}} \exp \left(-2 k_{t} \sqrt{\left(x-x_{0}\right)^{2}+\left(y-y_{0}\right)^{2}+\left(z-z_{0}\right)^{2}}\right) .
$$

In Figure $\mathrm{S} 9$ we show the result of the simulated $T_{1}$ map on Fe(TCNE) complex \#1. Since the tip height $z$ is a function of the lateral tip position $(x, y)$ due to the closed-loop topographic image (Figure S9(b)), we simulate the $T_{1}$ map utilizing the actual topography image as $z(x, y)$. We find that the best fit to the data is given by $x_{0}=-0.35 \mathrm{~nm}, y_{0}=$ $-0.40 \mathrm{~nm}$ (with respect to the center of the image, indicated by the red spot in Figure S9(b)) and $z_{0}=-0.32 \mathrm{~nm}$. The extracted relative distance between the tip and the spin center $(d)$ is shown in Figure $\mathrm{S} 9(\mathrm{c})$. The simulations show that $T_{1}$ is minimized $\left(r_{T_{1}}\right.$ is maximized) when the tip is closest to the spin center due to the enhanced tip-tip scattering (Figure S9(d)). The optimized value for the vertical distance of $z_{0}=-0.32 \mathrm{~nm}$ is also in good agreement with the point-contact distance of Fe on $2 \mathrm{ML}$ of $\mathrm{MgO}(\approx-0.28 \mathrm{~nm}$ or the given tunnel parameters: $10 \mathrm{mV}$ and $27 \mathrm{pA}$ as shown in Ref. 20). Therefore, the position of the spin center $\left(x_{0}, y_{0}\right)$ can be precisely identified from these $T_{1}$ map measurements. 
(a)

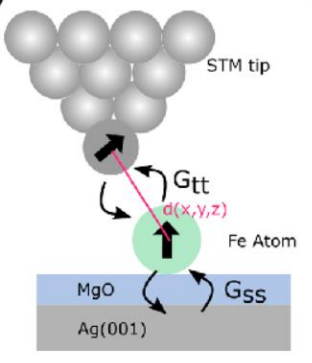

(b)

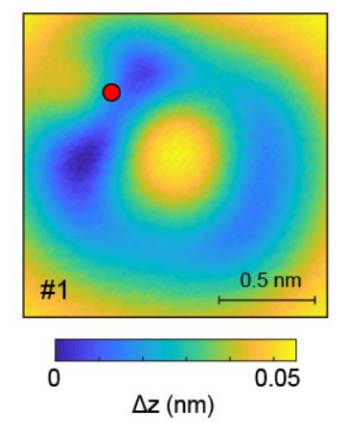

(c)

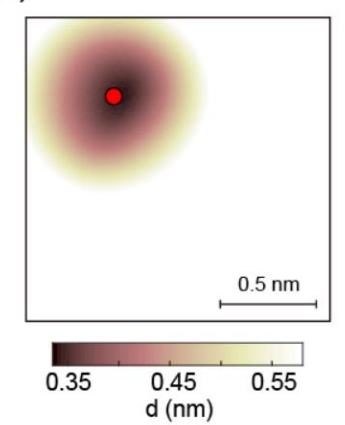

(d)

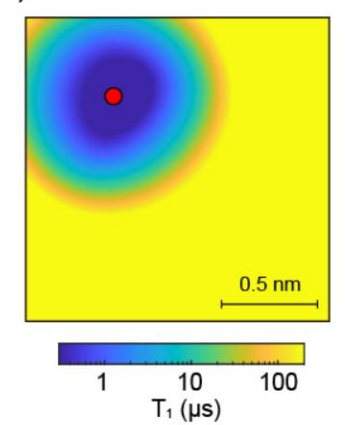

Figure S9. (a) Sketch of the experiment, indicating the sample-sample conductance $G_{S S}$, the tip-tip conductance $G_{t t}$ and the distance $d(x, y, z)$ between the tip apex and the Fe atom. (b) Topography of the $\mathrm{Fe}(\mathrm{TCNE})$ complex \#1. The position of the Fe spin center from the simulation is additionally indicated (red dot). (c) Distance $d$ between tip apex and the Fe spin center deduced from $d=$ $\sqrt{\left(x-x_{0}\right)^{2}+\left(y-y_{0}\right)^{2}+\left(z-z_{0}\right)^{2}}$. (d) Simulated $T_{1}$ map according to Eq. (S4).

\section{Details of DFT calculations}

All calculations were carried out in Quantum Espresso (version 6.4.1) and projector augmented wave (PAW) pseudopotentials from the PSL library with a cutoff for the wavefunction and charge density of 60 Ry and 600 Ry were used, respectively. ${ }^{34-36}$ Dispersive forces were treated within the Grimme-D3 van der Waals correction and periodic cells were decoupled using a dipole correction ${ }^{39,40}$. Calculations regarding the local electronic structure of Fe:3d were cross-checked with results obtained with a Hubbard-U correction ${ }^{41}$ for Fe which is known to be necessary for accurate description of the more localized $3 d$ orbitals, ${ }^{42}$ but no significant difference besides a rigid energy shift for the $3 d$ orbitals was found for values of $\mathrm{U}$ up to $5 \mathrm{eV}$ and therefore a Hubbard correction on Fe was not used for the remainder of the calculations.

The experimental setup is represented by $4 \times 4$ in-plane supercell of $2 \mathrm{ML} \mathrm{MgO}$ padded by 20 Angstrom of vacuum in the $z$-direction. This results in a lateral separation of at least 12 Angstrom between the complex and its periodic images. We performed calculations with and without $5 \mathrm{ML}$ of silver and found no difference in the local electronic structure of the complex which lead us to believe that $2 \mathrm{ML}$ of $\mathrm{MgO}$ effectively decouples the complex from the metal support. The silver layer was therefore removed in consecutive calculations. To obtain equilibrium geometries, the structures were relaxed with the lowest row of $\mathrm{MgO}$ frozen until the residual forces were less than $0.001 \mathrm{Ry} / \mathrm{a}_{0}$ which resulted in a Fe-O bond length of $0.18 \mathrm{~nm}$ and a distance between the $\mathrm{N}$ atoms of TCNE and the MgO surface of $0.28 \mathrm{~nm}$. The TCNE molecule appears slightly warped when fully relaxed as it accommodates the slight mismatch between $\mathrm{MgO}$ (length of O-O bond: $0.42 \mathrm{~nm}$ ) and the fully relaxed N-N distance of TCNE in vacuum (length of N-N bond: $0.44 \mathrm{~nm}$ ).

Figure S10 shows the setup with superimposed spin-polarization isosurface plotted using OVITO. ${ }^{43}$ The isosurface is plotted at a low value of $10^{-4}$ which emphasizes the 4-fold geometry of the oxygen top absorption site for Fe. The equilibrium lateral distance between Fe and TCNE is about 0.42 to $0.43 \mathrm{~nm}$ as shown in Figure S11. 

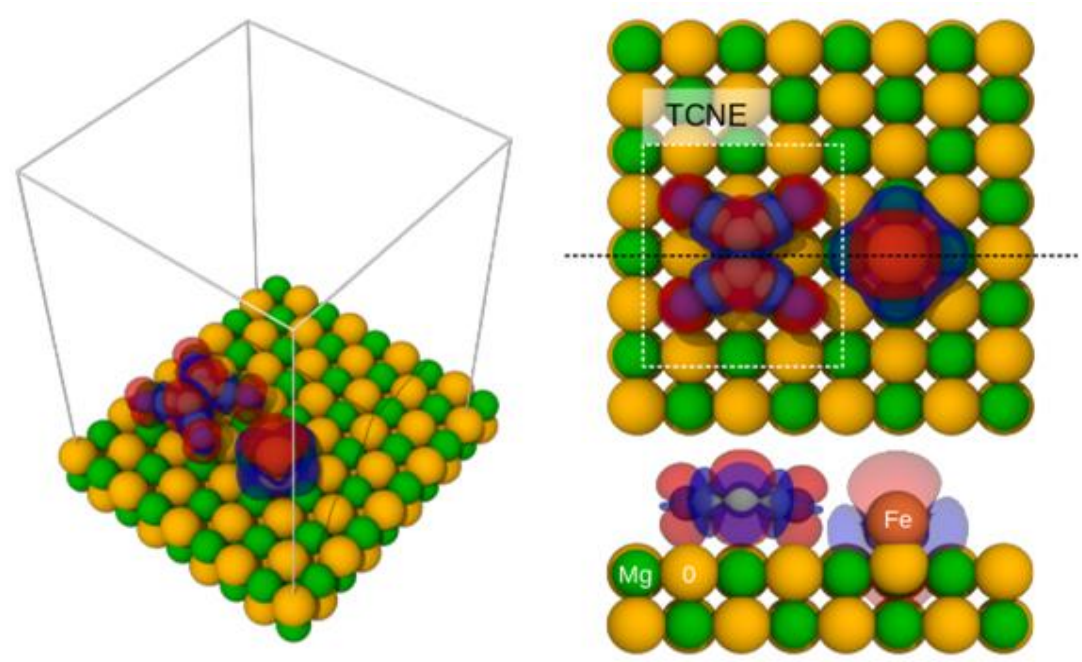

Figure S10. DFT calculation setup of the TCNE-Fe complex in "far" configuration showing spinpolarization $\left(\rho_{\uparrow}-\rho_{\downarrow}\right)$ isosurfaces plotted at an isosurface value of $10^{-4}$. Red (blue) corresponds to positive (negative) values of the spin-polarization. A top view and cross-sectional view along the dashed line are shown on the right.

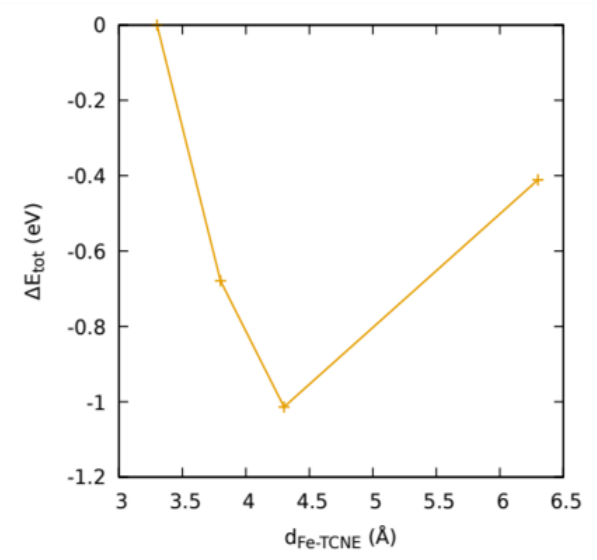

Figure S11. Total energy difference as function of the lateral Fe-TCNE separation.

Experimentally it was found that the TCNE molecule easily switches between several configurations, as shown in Figure S12. This indicates that the Fe-TCNE interaction is not strong or not directional which we can confirm by a series of calculations for different angles of rotation as indicated in Figure S13. The total barrier height is of $\sim 100 \mathrm{meV}$ between rotated states that look like saddle points (e.g. between 5 and 15 degrees the barrier becomes flat). An analysis of the electronic structure of these configurations indicate that the Fe: $3 d$ manifold is basically independent of shifts and rotations of the TCNE molecule unless the latter is brought into extreme proximity ( $<1 \mathrm{~nm}$ lateral separation) upon which the TCNE prefers to stand on top of the Fe. This configuration was never observed in measurements and therefore ignored. 
(a)

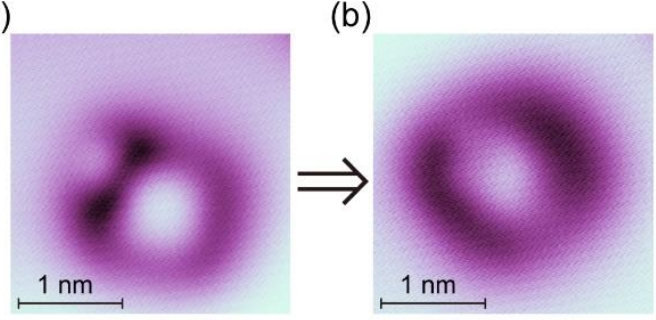

Figure S12. Molecule switching between different configurations. Scanning parameters: constantcurrent mode, $V=30 \mathrm{mV}, I=80 \mathrm{pA}$.

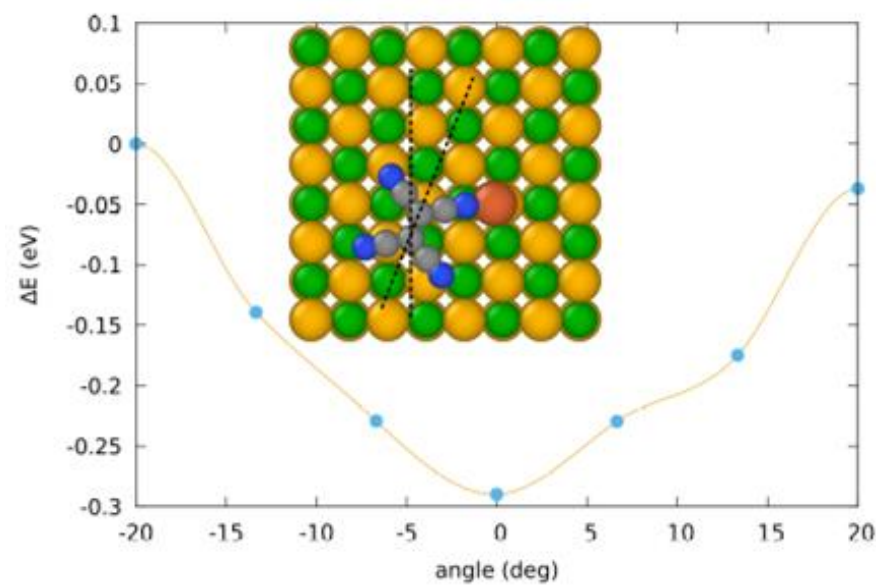

Figure S13. Total energy of the TCNE-Fe complex as function of the rotation angle of TCNE (indicated in the inset with 0 degree being the vertical axis). The barriers between rotated states are of the order roughly $50 \sim 100 \mathrm{meV}$.

To investigate the possibility of bond formation between Fe and TCNE we calculated the binding energy by total energy differences. Calculations indicate that the only bond formation is between $\mathrm{Fe}$ and $\mathrm{O}$ (binding energy $E_{\mathrm{B}} \sim 2.5 \mathrm{eV}$ ). The interaction energy for TCNE on $\mathrm{MgO}$ $(\sim 0.3 \mathrm{eV})$ and between TCNE and $\mathrm{Fe}(\sim 0.3 \mathrm{eV})$ is too low to indicate a bond formation and further does not show a strong directional dependence (see also Figure S13). This indicates that their respective interactions are mostly dispersive.

From the DFT calculations we were able to confirm that the TCNE-Fe interaction is weak and not strongly directional which explains the easy switching between configurations during the STM experiment. For all tested configurations the local electronic structure of the Fe:3d manifold was basically unchanged which supports the experimental observations of unchanged IETS and extrapolated $T_{1}$ of millisecond range.

\section{References}

[19] Loth, S.; Etzkorn, M.; Lutz, C. P.; Eigler, D. M.; Heinrich, A. J. Measurement of Fast Electron Spin Relaxation Times with Atomic Resolution. Science 2010, 329, 1628-1630.

[20] Paul, W.; Yang, K.; Baumann, S.; Romming, N.; Choi, T.; Lutz, C. P.; Heinrich, A. J. Control of the Millisecond Spin Lifetime of An Electrically Probed Atom. Nat. Phys. 2017, 13, 403-407. 
[34] Giannozzi, P.; Baroni, S.; Bonini, N.; Calandra, M.; Car, R.; Cavazzoni, C.; Ceresoli, D.; Chiarotti, G. L.; Cococcioni, M.; Dabo, I.; Corso, A. D.; de Gironcoli, S.; Fabris, S.; Fratesi, G.; Gebauer, R.; Gerstmann, U.; Gougoussis, C.; Kokalj, A.; Lazzeri, M.; Martin-Samos, L. et al. QUANTUM ESPRESSO: A Modular and Open-Source Software Project for Quantum Simulations of Materials. J. Phys.: Condens. Matter 2009, 21, 395502.

[35] Giannozzi, P.; Andreussi, O.; Brumme, T.; Bunau, O.; Nardelli, M. B.; Calandra, M.; Car, R.; Cavazzoni, C.; Ceresoli, D.; Cococcioni, M.; Colonna, N.; Carnimeo, I.; Corso, A. D.; de Gironcoli, S.; Delugas, P.; DiStasio Jr, R. A.; Ferretti, A.; Floris, A.; Fratesi, G.; Fugallo, G. et al. Advanced Capabilities for Materials Modelling with Quantum ESPRESSO. J. Phys.: Condens. Matter 2017, 29,465901

[36] Dal Corso, A. Pseudopotentials Periodic Table: From H to Pu. Comput. Mater. Sci. 2014, 95, 337-350.

[37] Loth, S.; von Bergmann, K.; Ternes, M., Otte, A. F.; Lutz, C. P.; Heinrich, A. J. Controlling the State of Quantum Spins with Electric Currents. Nat. Phys. 2010, 6, 340-344.

[38] Willke, P.; Singha, A.; Zhang, X.; Esat, T.; Lutz, C. P.; Heinrich, A. J.; Choi, T. Tuning SingleAtom Electron Spin Resonance in a Vector Magnetic Field. Nano Lett. 2019, 19, 8201-8206.

[39] Grimme, S.; Hansen, A.; Brandenburg, J. G.; Bannwarth, C. Dispersion-Corrected Mean-Field Electronic Structure Methods. Chem. Rev. 2016, 116, 5105-5154.

[40] Bengtsson, L. Dipole Correction for Surface Supercell Calculations. Phys. Rev. B 1999, 59, 12301-12304.

[41] Cococcioni, M.; de Gironcoli, S. Linear Response Approach to the Calculation of the Effective Interaction Parameters in the LDA+U Method. Phys. Rev. B 2005, 71, 035105.

[42] Linscott, E. B.; Cole, D. J.; Payne, M. C.; O'Regan, D. D. Role of Spin in the Calculation of Hubbard $U$ and Hund's $J$ Parameters from First Principles. Phys. Rev. B 2018, 98, 235157.

[43] Stukowski, A. Visualization and Analysis of Atomistic Simulation Data with OVITO-the Open Visualization Tool. Modelling Simul. Mater. Sci. Eng. 2010, 18, 015012. 